\title{
In utero hematopoietic cell transplantation: induction of donor specific immune tolerance and postnatal transplants
}

\author{
William H. Peranteau* \\ Department of Surgery, Center for Fetal Research, The Children's Hospital of Philadelphia, Philadelphia, PA, USA
}

Edited by:

Tippi C. MacKenzie, University of California, San Francisco, USA

\section{Reviewed by:}

Graca Almeida-Porada, Wake Forest Institute for Regenerative Medicine, USA

Christopher D. Porada, Wake Forest Institute for Regenerative Medicine, USA

\section{${ }^{*}$ Correspondence:}

William H. Peranteau, Department of Surgery, Center for Fetal Research, The Children's Hospital of Philadelphia 3615 Civic Center Boulevard,

Abramson Research Center 1116E, Philadelphia, PA 19104, USA

e-mail:peranteauw@email.chop.edu
In utero hematopoietic cell transplantation (IUHCT) is a non-myeloablative nonimmunosuppressive transplant approach that allows for donor cell engraftment across immunologic barriers. Successful engraftment is associated with donor-specific tolerance. IUHCT has the potential to treat a large number of congenital hematologic, immunologic, and genetic diseases either by achieving high enough engraftment levels following a single IUHCT or by inducing donor specific tolerance to allow for non-toxic same-donor postnatal transplants. This review evaluates donor specific tolerance induction achieved by IUHCT. Specifically it addresses the need to achieve threshold levels of donor cell engraftment following IUHCT to consistently obtain immunologic tolerance. The mechanisms of tolerance induction including partial deletion of donor reactive host $T$ cells by direct and indirect antigen presentation and the role of regulatory $T$ cells in maintaining tolerance are reviewed. Finally, this review highlights the promising clinical potential of in utero tolerance induction to provide a platform on which postnatal cellular and organ transplants can be performed without myeloablative or immunosuppressive conditioning.

Keywords: in utero, immune tolerance, postnatal transplant, fetus, hematopoietic stem cell, myeloablation, immunosuppression, in utero hematopoietic cell transplantation

\section{INTRODUCTION}

The fetal environment offers the unique opportunity to take advantage of the developing immune system to induce immunologic tolerance to foreign antigen. This was initially recognized in an experiment of nature in which Owen observed permanent red blood cell chimerism in dizygotic cattle twins that shared cross-placental circulation (Owen, 1945). Later studies by Billingham, Medawar, and others confirmed the ability to induce immunologic tolerance by early gestational exposure to foreign antigen (Anderson et al., 1951; Billingham etal., 1952; Simonsen, 1955). In utero hematopoietic cell transplantation (IUHCT) seeks to take advantage of this developmental phenomenon. In multiple animal models, IUHCT has been shown to be a non-myeloablative nonimmunosuppressive transplant approach that allows for engraftment across immunologic barriers and is associated with the induction of donor specific tolerance (Flake and Zanjani, 1999; Kim etal., 1999; Peranteau et al., 2002). Clinically, IUHCT has the potential to treat any congenital hematologic, genetic or immunologic disorder which can be prenatally diagnosed and which is currently managed with a postnatal hematopoietic stem cell (HSC) transplantation requiring a matching donor and/or myeloabalative and immunosuppressive conditioning.

The clinical application of IUHCT could take one of two potential courses (Figure 1). A single in utero transplant may result in high enough levels of donor cell engraftment to treat the target disease. Alternatively, IUHCT may be used to induce donor specific tolerance which would allow for postnatal same-donor transplants with non-toxic conditioning regimens to increase donor cell engraft to clinically relevant levels. Tolerance achieved by IUHCT may also be used to permit postnatal same-donor organ transplants without immunosuppressive conditioning. To date, IUHCT has only been clinically successful in the treatment of severe combined immunodeficiency disorder (SCID; Flake et al., 1996; Wengler et al., 1996). Broader clinical application of IUHCT is limited by the ability to consistently achieve high enough levels of donor cell engraftment to treat the target disease. Thus, tolerance induction by IUHCT to allow for postnatal "booster" transplants may be instrumental to the future clinical application of IUHCT. In this review, we focus on the progress that has been made in understanding and achieving immunologic tolerance following IUHCT and how this tolerance can be used as a platform for non-myeloablative non-immunosuppressive postnatal transplants to either achieve clinically acceptable levels of engraftment or allow for solid organ transplants.

\section{IUHCT AND ALLOGENEIC ENGRAFTMENT: FROM MICRO TO MACROCHIMERISM AND TOLERANCE INDUCTION}

In utero hematopoietic cell transplantation has been studied in multiple animal models. Initial results in the sheep model were very encouraging demonstrating stable long-term hematopoietic chimerism in three of four sheep following IUHCT (Flake et al., 1986). Unfortunately, these findings did not translate into similar results in clinical studies. Successful engraftment following IUHCT in humans has been limited to circumstances of immunodeficiency and those in which a donor cell selective advantage exists (Flake etal., 1996; Wengler etal., 1996; Gil et al., 1999; Bartolome et al., 2002; Pirovano et al., 2004; Touraine et al., 2004; 


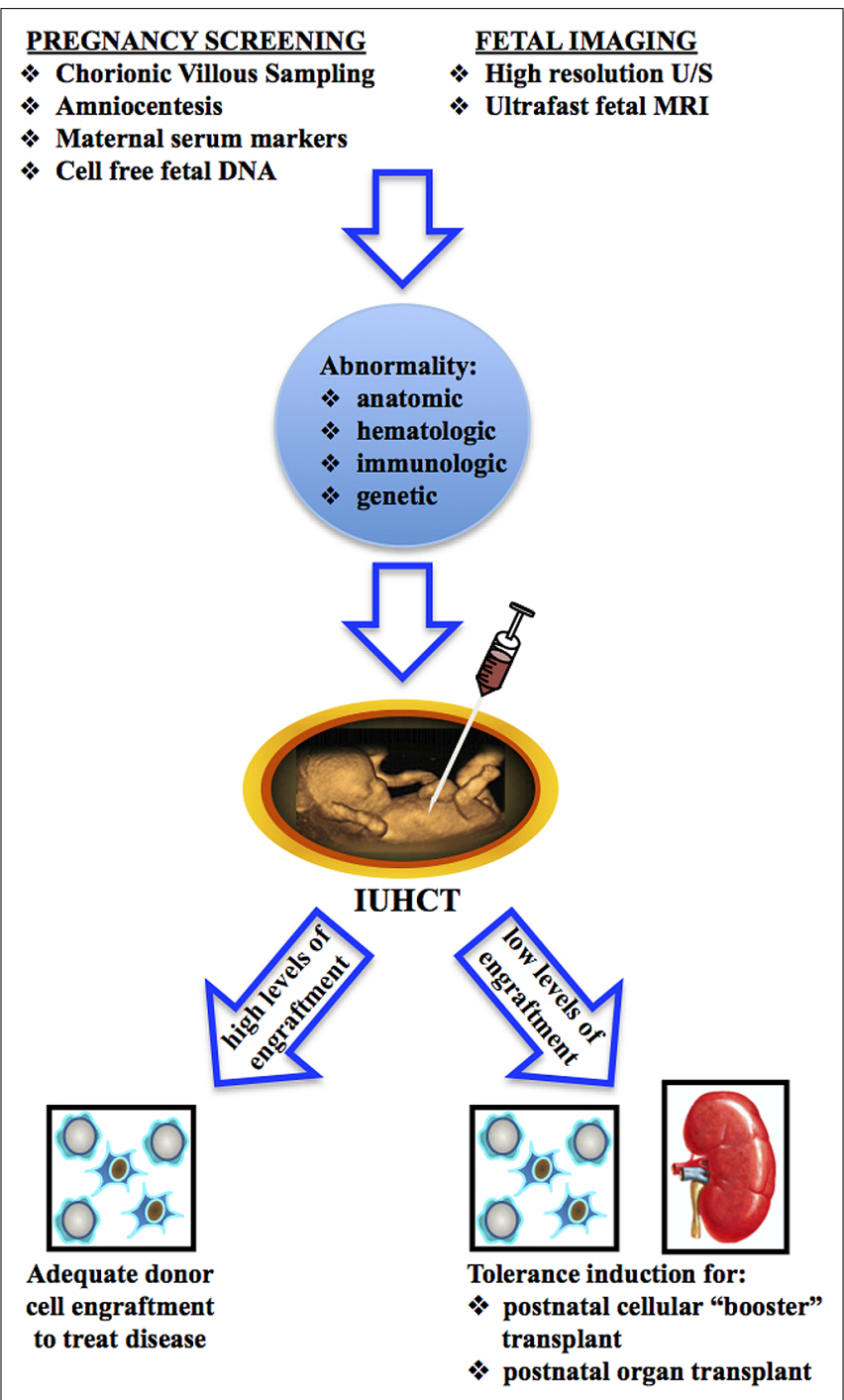

FIGURE 1 | Two approaches for the clinical application of IUHCT.

Muench, 2005; De Santis et al., 2011). These discouraging results highlighted the need for a more in depth study of the events following IUHCT including the induction of donor specific tolerance. To this aim, murine models of IUHCT have been developed. Studies in these models support an intimate relationship between the levels of donor cell chimerism following IUHCT and tolerance induction. In chimeric mice in which donor cell engraftment was only detectable by PCR and undetectable by flow cytometry (microchimerism), donor specific tolerance, as demonstrated by skin graft acceptance, response to postnatal boosting transplants, and in vitro proliferation assays, is inconsistent and occurs in only a subset of animals (Billingham et al., 1953; Carrier et al., 1995; Kim et al., 1999). Interestingly, studies in mice and large animals have found that tolerance following IUHCT may persistent even when peripheral blood chimerism levels are low if donor cells persist in tissues or the peritoneal cavity of recipients (Carrier et al., 1995; Mathes et al., 2001, 2005; Chen et al., 2004). Technical advances, including the ability to deliver higher doses of donor cells at the time of IUHCT via an intravenous injection, have allowed for the creation of mice with chimerism levels consistently greater than $1 \%$ (macrochimerism; Peranteau et al., 2006, 2007). The ability to achieve higher initial levels of donor cell engraftment has demonstrated that induction of donor specific tolerance can be consistently achieved in macrochimeric animals and tolerance correlates with donor chimerism levels (Hayashi et al., 2002; Ashizuka et al., 2006). Specifically, 60\% of mice with peripheral blood chimerism levels of less than $1 \%$, and $100 \%$ of mice with chimerism levels greater than $1 \%$ following IUHCT demonstrated successful enhancement of allogeneic engraftment following postnatal, same-donor, bone marrow (BM) transplants suggestive of the presence of donor specific tolerance. Decreased donor specific reactivity was demonstrated by MLR in those mice with $<1 \%$ chimerism in which engraftment could be successfully enhanced following IUHCT compared to those in which engraftment could not be enhanced (Ashizuka et al., 2006). In another study, peripheral blood chimerism was noted to correlate with thymic chimerism and donor specific tolerance as measured by skin graft acceptance. In this study, chimerism levels greater than $3 \%$ at the time of skin graft placement were consistently associated with donor specific tolerance and graft acceptance (Chen et al., 2010). In this study, adequate levels of donor cell engraftment were needed for the induction of tolerance. However, peripheral blood chimerism was not required for the maintenance of tolerance as demonstrated by persistence of donor skin grafts despite the loss of peripheral blood chimerism in some mice.

\section{IUHCT AND MECHANISM OF DONOR SPECIFIC TOLERANCE}

Fetal immunologic tolerance is a phenomenon believed to be temporally related to thymic development (Billingham et al., 1953). The developing fetal thymic microenvironment plays a primary role in the positive and negative selection of pre-T cells resulting in the deletion of presumed auto-reactive $\mathrm{T}$ cell clones with a high affinity for self antigen in association with self MHC while maintaining a T cell repertoire for foreign antigen (Sprent, 1995; Goodnow, 1996; Goodnow et al., 2005). In the human fetus, TCR bearing, single positive lymphocytes can be identified as early as 13-14 weeks gestation. In the murine system, this stage of development corresponds to $\sim 17$ days gestation. Thus, IUHCT attempts to introduce donor cells into the fetal thymic microenvironment prior to this time such that donor cells will be identified as "self" and donor antigens will undergo appropriate thymic antigen presentation resulting in clonal deletion of donor alloreactive host $\mathrm{T}$ cells.

Although donor specific tolerance following IUHCT is well accepted, the mechanisms underlying this tolerance have only recently begun to be understood. Early studies suggested tolerance was the result of partial deletion of donor specific host $\mathrm{T}$ cells combined with peripheral suppression of donor reactive $\mathrm{T}$ cells that escape deletion (Kim et al., 1999; Nijagal et al., 2011). Thymic deletion of donor reactive host $\mathrm{T}$ cells can occur via the direct pathway in which donor antigen is presented by donor antigen presenting cells (APCs) or the indirect pathway in which recipient APCs process donor derived allo-MHC molecules into peptides and then present those peptides to T-cells on self-class II 
MHC molecules. Additionally, the "semidirect" pathway whereby intact donor MHC molecule-donor peptide complexes are taken up by host APCs and directly interact with reactive T cells may be involved (Herrera et al., 2004; Nijagal et al., 2013). Initial studies using the mammary tumor virus $(M t v)$ superantigen system demonstrated that partial deletion of donor reactive host lymphocytes occurs via both the indirect and direct route of antigen presentation following IUHCT (Shaaban et al., 2000; Peranteau et al., 2002). More recently, murine studies using TCR-transgenic systems that allow differentiation of direct vs. indirect antigen presentation with subsequent donor reactive $\mathrm{T}$ cell deletion confirm that deletional tolerance can occur via both pathways (Nijagal et al., 2013). In this study, expression of donor-derived class II antigens on host APCs was assessed to determine the possible contribution of the "semidirect" pathway to deletion of donor reactive T cells. No expression was seen suggesting that the "semidirect" pathway does not play a significant role in deletional tolerance following IUHCT. In addition to inducing immunologic tolerance of host cells to donor cells, IUHCT also results in partial deletion of host reactive donor $\mathrm{T}$ cells derived from hematopoietic stem or early progenitor cells at the time of IUHCT via the direct pathway (Bacchetta etal., 1993; Shaaban et al., 2000; Peranteau et al., 2002). In these studies, the direct route of antigen presentation was more efficient with respect to the degree of relevant clonal deletion, but neither route resulted in complete deletion of donor (or host) reactive lymphocytes. Remaining donor (or host) reactive lymphocytes are thought to be suppressed in the periphery by mechanisms that remain to be fully elucidated. This is similar to clinical experience in children who have undergone a successful IUHCT for SCID. These children were immunologically tolerant and were shown to have residual clones of donor reactive cells that were anergic in proliferative assays (Roncarolo et al., 1988; Sakaguchi et al., 1995; Touraine et al., 2005). The mechanism by which this occurs is hypothesized to be related to peripheral regulatory cells using the natural mechanisms of controlling autoreactive T cells that escape thymic deletion (Muench, 2005). In the murine model, the contribution of $\mathrm{CD} 4{ }^{+} \mathrm{CD} 25^{+} \mathrm{Foxp}^{+}$ $\mathrm{T}$ regulatory cells to this process remains unclear. Studies have shown an increase in the percentage of Treg cells (as well as the Treg/Teff ratio) in the thymus and spleen of chimeric mice following IUHCT related to deletion of the Teff population but not an increase in the absolute number of Treg cells (Nijagal et al., 2013). Although this shift in the Treg/Teff ratio may play an important role in the establishment of engraftment, the contribution of Tregs to maintaining chimerism following IUHCT remains to be shown.

\section{IN UTERO TOLERANCE INDUCTION AND POSTNATAL TRANSPLANTS}

Technical improvements in injection techniques have highlighted the intravascular route as a promising alternative to the intraperitoneal route of injection. IUHCT via the intravascular route has achieved initial chimerism levels of 1-23 and 3-39\% in the murine model and the preclinical canine model respectively (Peranteau et al., 2006; Vrecenak et al., 2014). These results are encouraging and those animals at the higher end of the engraftment spectrum have donor cell chimerism levels that may be adequate to treat the target disease. However, studies in murine models of Sickle cell anemia, a primary target disease for treatment by IUHCT, suggest that 70 and $40 \%$ donor cell myeloid engraftment is needed to eliminate peripheral RBC sickling and anemia respectively (Iannone et al., 2001). To obtain these and higher levels of engraftment in all recipients of IUHCT, alternative approaches must be explored. Ex vivo modification of donor HSCs or in vivo treatment of fetal recipients with agents which provide a competitive advantage to donor HSCs over endogenous fetal HSCs may be employed to increase donor cell engraftment to clinically relevant levels following a single IUHCT (Peranteau et al., 2006; Derderian et al., 2014). Alternatively, donor specific tolerance induction by IUHCT can be used as a platform on which postnatal transplants using the same prenatal donor source can be performed following nonmyeloablative, non-immunosuppressive conditioning to increase engraftment levels.

A review of the literature reveals multiple studies demonstrating the feasibility of tolerance induction by IUHCT followed by postnatal same-donor "booster" transplants (Table 1). In the murine model, allogeneic donor cell engraftment was minimally increased when postnatal same-donor transplants were performed in the absence of any conditioning regimen (Carrier et al., 1995; Donahue et al., 2001). We performed additional studies in which non-myeloablative non-toxic conditioning regimens, including low dose total body irradiation (TBI) or busulfan, were administered to chimeric recipients prior to a postnatal samedonor transplant (Peranteau etal., 2002; Ashizuka et al., 2006). Engraftment enhancement directly correlated with the dose of TBI or busulfan administered with near complete donor cell chimerism achieved at the highest doses. The increase in donor cell chimerism resulted from the postnatal donor cell source as opposed to expansion of donor HSCs which had engrafted following IUHCT. Finally, chimeric mice in which engraftment was successfully enhanced demonstrated reduced donor cell reactivity of recipient cells by MLR following IUHCT and prior to the postnatal transplant. These studies highlight the potential to increase allogeneic donor cell engraftment to clinically relevant levels by a combination of tolerance induction by IUHCT and engraftment enhancement by a postnatal BMT using the same prenatal donor. They demonstrate the need for some conditioning regimen to provide a competitive advantage to the donor cell population to achieve the desired engraftment levels independent of preexisting immunologic tolerance. Studies in the preclinical canine model also support the feasibility of this approach with results that reflect similar findings to those achieved in the murine model. Specifically, we demonstrated the ability to successfully enhance peripheral blood donor cell chimerism in the canine model by a combination of IUHCT and a postnatal same-donor BMT using a low-dose busulfan conditioning regimen (Peranteau etal., 2009). In this study, donor chimerism levels were increased from $<1$ to $35-50 \%$ and remained stable up to 6 months to 1 year after transplant in two of six recipients. Control dogs which did not receive an IUHCT never demonstrated any donor cell engraftment following postnatal BMT. The 33\% success rate of enhancing engraftment in dogs with initial chimerism levels $<1 \%$ following IUHCT concurs with murine studies in which $60 \%$ of mice with chimerism levels $<1 \%$ 
Table 1 | Summary of studies using IUHCT to induce donor specific tolerance for postnatal allogeneic cellular or organ transplants.

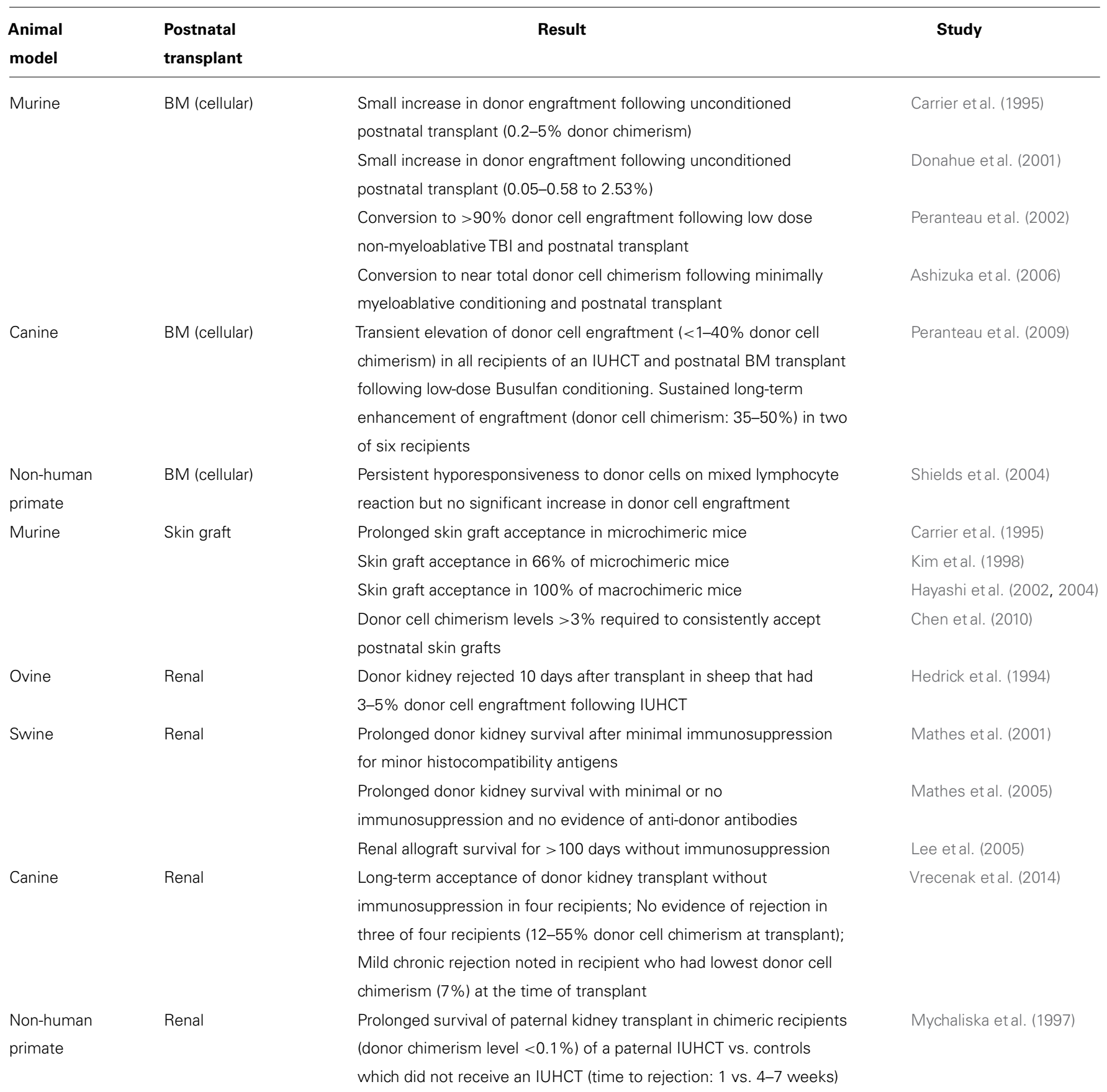

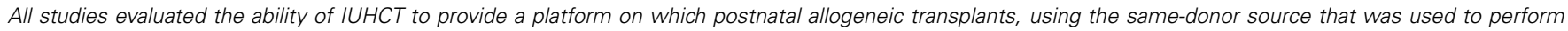

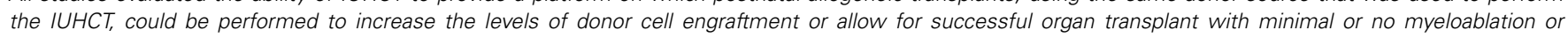
immunosuppression.

BM, bone marrow; TBI, total body irradiation.

following IUHCT successfully enhanced donor cell engraftment using a similar postnatal transplant regimen (Ashizuka et al., 2006). In both studies, failure to achieve stable enhanced donor cell engraftment was associated with increased donor cell reactivity of recipient cells on MLR suggesting a lack of definitive tolerance. These studies support the need to achieve initial levels of donor cell engraftment $>1 \%$ following IUHCT to reliably induce donor specific tolerance for postnatal cellular transplants. More recently, IUHCT via the intracardiac route in the canine model has more consistently resulted in donor cell engraftment at levels believed to be associated with donor specific tolerance (Vrecenak etal., 2014). These results highlight the potential to 
more reliably enhance donor cell chimerism by the combination of IUHCT and postnatal same-donor transplants in the clinical setting.

In utero hematopoietic cell transplantation may also induce donor specific tolerance and allow for postnatal solid organ transplants without the requirement for immunosuppressive conditioning (Table 1). Acceptance of donor skin grafts, a classic method of assessing donor specific tolerance, has been repeatedly demonstrated in the murine model of IUHCT with success dependent on the levels of donor cell chimerism (Chen et al., 2010). A renal transplant is potentially the most clinically relevant solid organ transplant in the setting of tolerance induction by IUHCT. Studies in the swine and canine model support the ability of donor specific tolerance induction by IUHCT to allow for successful postnatal same-donor renal transplants without immunosuppression. Interestingly, in the canine model, clinically insignificant but histologically detected mild chronic rejection of one recipient of a postnatal renal transplant following IUHCT was noted. This recipient had the lowest levels of peripheral blood donor cell chimerism (7\%) at the time of renal transplant. The other renal transplant recipients had chimerism levels of $12-55 \%$ at the time of transplant and demonstrated no clinical or histologic evidence of rejection (Vrecenak et al., 2014). Donor cell engraftment levels of $7 \%$ are above what would be expected to induce donor cell tolerance and allow for successful non-myeloablative postnatal cellular transplants suggesting that chimerism levels that allow for successful postnatal solid organ transplants without immunosuppression may be different than those required for postnatal cellular transplants. Finally, tolerance induction by IUHCT to allow for xenogeneic solid organ transplants has also been investigated. Results from these limited studies highlight the potential of this approach to overcome the immune limitation to xenogeneic transplantation (Tanaka et al., 1998).

\section{CONCLUSION}

In utero hematopoietic cell transplantation is a non-myeloablative non-immunosuppressive transplant approach that allows for donor cell engraftment and donor specific tolerance across immunologic barriers. It has the potential to treat a large number of congenital hematologic, genetic, and immunologic disorders which, because of advances in prenatal care, can be diagnosed before birth and before the maturation of the fetal immune system. Studies in murine and preclinical large animal models suggest that, in limited circumstances, a single IUHCT may result in high enough levels of donor cell engraftment to ameliorate the target disease. However, even in the absence of obtaining therapeutic levels of engraftment, the major benefit of IUHCT may be in the reliable induction of donor specific tolerance to allow for postnatal non-myeloablative same-donor cellular transplants to enhance engraftment to target levels with minimal treatment related toxicity. Although less clinically relevant at the current time, a similar approach of tolerance induction by IUHCT to allow for postnatal organ transplants without immunosuppression may hold promise in the future. In order to embrace the full potential of in utero tolerance induction for postnatal cellular and organ transplants, additional insights into the mechanisms involved in the induction and maintenance of tolerance including the role of peripheral regulatory cells as well as the barriers to engraftment that prevent the acquisition of donor specific tolerance in all recipients of IUHCT must be investigated.

\section{REFERENCES}

Anderson, D., Billingham, R. E., Lampkin, G. H., and Medawar, P. B. (1951). The use of skin grafts to distinguish between monozygotic and dizygotic twins in cattle. Heredity (Edinb.) 5, 379-397. doi: 10.1038/hdy.1951.38

Ashizuka, S., Peranteau, W. H., Hayashi, S., and Flake, A. W. (2006). Busulfan-conditioned bone marrow transplantation results in high-level allogeneic chimerism in mice made tolerant by in utero hematopoietic cell transplantation. Exp. Hematol. 34, 359-368. doi: 10.1016/j.exphem.2005.1 1.011

Bacchetta, R., Vandekerckhove, B. A., Touraine, J. L., Bigler, M., Martino, S., Gebuhrer, L., et al. (1993). Chimerism and tolerance to host and donor in severe combined immunodeficiencies transplanted with fetal liver stem cells. J. Clin. Invest. 91, 1067-1078. doi: 10.1172/JCI116264

Bartolome, J., Porta, F., Lafranchi, A., Rodriguez-Molina, J. J., Cela, E., Cantalejo, A., et al. (2002). B cell function after haploidentical in utero bone marrow transplantation in a patient with severe combined immunodeficiency. Bone Marrow Transplant. 29, 625-628. doi: 10.1038/sj.bmt.1703410

Billingham, R., Brent, L., and Medawar, P. B. (1953). Activity acquired tolerance of foreign cells. Nature 172, 603-606. doi: 10.1038/172603a0

Billingham, R. E., Lampkin, G. H., Medawar, P. B., and Williams, H. L. L. (1952). Tolerance to homografts, twin diagnosis, and the freemartin condition in cattle. Heredity (Edinb.) 6, 201-212. doi: 10.1038/hdy.1952.20

Carrier, E., Lee, T. H., Busch, M. P., and Cowan, M. J. (1995). Induction of tolerance in nondefective mice after in utero transplantation of major histocompatibility complex-mismatched fetal hematopoietic stem cells. Blood 86, 4681-4690.

Chen, J. C., Chang, M. L., Lee, H., and Muench, M. O. (2004). Haploidentical donor $\mathrm{T}$ cells fail to facilitate engraftment but lessen the immune response of host $\mathrm{T}$ cells in murine fetal transplantation. Br. J. Haematol. 126, 377-384. doi: 10.1111/j.1365-2141.2004.05040.x

Chen, J. C., Kuo, M. L., Ou, L. S., Chang, P. Y., Muench, M. O., Shen, C. R., et al. (2010). Characterization of tolerance induction through prenatal marrow transplantation: the requirement for a threshold level of chimerism to establish rather than maintain postnatal skin tolerance. Cell Transplant. 19, 1609-1622. doi: $10.3727 / 096368910 \times 516583$

Derderian, S. C., Togarrati, P. P., King, C., Moradi, P. W., Reynaud, D., Czechowicz, A., et al. (2014). In utero depletion of fetal hematopoietic stem cells improves engraftment after neonatal transplantation in mice. Blood 124, 973-980. doi: 10.1182/blood-2014-02-550327

De Santis, M., De Luca, C., Mappa, I., Cesari, E., Quattrocchi, T., Spagnuolo, T., et al. (2011). In-utero stem cell transplantation: clinical use and therapeutic potential. Minerva Ginecol. 63, 387-398. doi: 10.1016/j.jcyt.2013.01.003

Donahue, J., Gilpin, E., Young, D., and Carrier, E. (2001). Postnatal cytokines and boosts improve chimerism and hematological parameters in betathalassemic mice transplanted in utero. Transplantation 71, 1491-1494. doi: 10.1097/00007890-200105270-00025

Flake, A. W., Harrison, M. R., Adzick, N. S., and Zanjani, E. D. (1986). Transplantation of fetal hematopoietic stem cells in utero: the creation of hematopoietic chimeras. Science 233, 776-778. doi: 10.1126/science.2874611

Flake, A. W., Roncarolo, M. G., Puck, J. M., Almeida-Porada, G., Evans, M. I., Johnson, M. P., et al. (1996). Treatment of X-linked severe combined immunodeficiency by in utero transplantation of paternal bone marrow. N. Engl. J. Med. 335, 1806-1810. doi: 10.1056/NEJM199612123352404

Flake, A. W., and Zanjani, E. D. (1999). In utero hematopoietic stem cell transplantation: ontogenic opportunities and biologic barriers. Blood 94, 2179-2191.

Gil, J., Porta, F., Bartolome, J., Lafranchi, A., Verardi, R., Notarangelo, L. D., et al. (1999). Immune reconstitution after in utero bone marrow transplantation in a fetus with severe combined immunodeficiency with natural killer cells. Transplant. Proc. 31:2581. doi: 10.1016/S0041-1345(99)00510-2

Goodnow, C. C. (1996). Balancing immunity and tolerance: deleting and tuning lymphocyte repertoires. Proc. Natl. Acad. Sci. U.S.A. 93, 2264-2271. doi: 10.1073/pnas.93.6.2264 
Goodnow, C. C., Sprent, J., Fazekas de St Groth, B., and Vinuesa, C. G. (2005). Cellular and genetic mechanisms of self tolerance and autoimmunity. Nature 435, 590-597. doi: 10.1038/nature03724

Hayashi, S., Hsieh, M., Peranteau, W. H., Ashizuka, S., and Flake, A. W. (2004). Complete allogeneic hematopoietic chimerism achieved by in utero hematopoietic cell transplantation and cotransplantation of LLME-treated, MHC-sensitized donor lymphocytes. Exp. Hematol. 32, 290-299. doi: 10.1016/j.exphem.2003.12.008

Hayashi, S., Peranteau, W. H., Shaaban, A. F., and Flake, A. W. (2002). Complete allogeneic hematopoietic chimerism achieved by a combined strategy of in utero hematopoietic stem cell transplantation and postnatal donor lymphocyte infusion. Blood 100, 804-812. doi: 10.1182/blood-2002-01-0016

Hedrick, M. H., Rice, H. E., MacGillivray, T. E., Bealer, J. F., Zanjani, E. D., and Flake, A. W. (1994). Hematopoietic chimerism achieved by in utero hematopoietic stem cell injection does not induce donor-specific tolerance for renal allografts in sheep. Transplantation 58, 110-111. doi: 10.1097/00007890-199407000-00020

Herrera, O. B., Golshaya, D., Tibbott, R., Lombardi, G., and Lechler, R. I. (2004). A novel pathway of alloantigen presentation by dendritic cells. J. Immunol. 173, 4828-4837. doi: 10.4049/jimmunol.173.8.4828

Iannone, R., Luznik, L., Engstrom, L. W., Tennessee, S. L., Askin, F. B., Casella, J. F., et al. (2001). Effects of mixed hematopoietic chimerism in a mouse model of bone marrow transplantation for sickle cell anemia. Blood 97, 3960-3965. doi: 10.1182/blood.V97.12.3960

Kim, H. B., Shaaban, A. F., Milner, R., Fichter, C., and Flake, A. W. (1999). In utero bone marrow transplantation induces donor-specific tolerance by a combination of clonal deletion and clonal anergy. J. Pediatr. Surg. 34, 726-730. doi: 10.1016/S0022-3468(99)90364-0

Kim, H. B., Shaaban, A. F., Yang, E. Y., Liechty, K. W., and Flake, A. W. (1998). Microchimerism and tolerance after in utero bone marrow transplantation in mice. J. Surg. Res. 77, 1-5. doi: 10.1006/jsre.1997.5255

Lee, P. W., Cina, R. A., Randolph, M. A., Arellano, R., Goodrich, J., Rowland, H., et al. (2005). In utero bone marrow transplantation induces kidney allograft tolerance across a full major histocompatibility complex barrier in swine. Transplantation 79, 1084-1090. doi: 10.1097/01.TP.0000161247.61727.67

Mathes, D. W., Solari, M. G., Randolph, M. A., Gazelle, G. S., Yamada, K., Huang, C. A., et al. (2005). Long-term acceptance of renal allografts following prenatal inoculation with adult bone marrow. Transplantation 80, 1300-1308. doi: 10.1097/01.tp.0000178933.31987.11

Mathes, D. W., Yamada, K., Randolph, M. A., Utsugi, R., Solari, M. G., Gazelle, G. S., et al. (2001). In utero induction of transplantation tolerance. Transplant. Proc. 33, 98-100. doi: 10.1016/S0041-1345(00)01924-2

Muench, M. O. (2005). In utero transplantation: baby steps towards an effective therapy. Bone Marrow Transplant. 35, 537-547. doi: 10.1038/sj.bmt.1704811

Mychaliska, G. B., Rice, H. E., Tarantal, A. F., Stock, P. G., Capper, J., Garovoy, M. R., et al. (1997). In utero hematopoietic stem cell transplants prolong survival of postnatal kidney transplantation in monkeys. J. Pediatr. Surg. 32, 976-981. doi: 10.1016/S0022-3468(97)90381-X

Nijagal, A., Derderian, C., Le, T., Jarvis, E., Nguyen, L., Tang, Q., et al. (2013). Direct and indirect antigen presentation lead to deletion of donor-specific $\mathrm{T}$ cells after in utero hematopoietic cell transplantation in mice. Blood 121, 4595-4602. doi: 10.1182/blood-2012-10-463174

Nijagal, A., Wegorzewska, M., Jarvis, E., Le, T., Tang, Q., and MacKenzie, T. (2011). Maternal T cells limit engraftment after in utero hematopoietic cell transplantation in mice. J. Clin. Invest. 121, 582-592. doi: 10.1172/JCI44907

Owen, R. D. (1945). Immunogenetic consequences of vascular anastomoses between bovine cattle twins. Science 102, 400-401. doi: 10.1126/science.102. 2651.400

Peranteau, W. H., Endo, M., Adibe, O. O., and Flake, A. W. (2007). Evidence for an immune barrier after in utero hematopoietic-cell transplantation. Blood 109, 1331-1333. doi: 10.1182/blood-2006-04-018606

Peranteau, W. H., Endo, M., Adibe, O. O., Merchant, A., Zoltick, P. W., and Flake, A. W. (2006). CD26 inhibition enhances allogeneic donor-cell homing and engraftment after in utero hematopoietic-cell transplantation. Blood 108, 4268-4274. doi: 10.1182/blood-2006-04-018986

Peranteau, W. H., Hayashi, S., Hsieh, M., Shaaban, A. F., and Flake, A. W. (2002). High-level allogeneic chimerism achieved by prenatal tolerance induction and postnatal nonmyeloablative bone marrow transplantation. Blood 100, 2225-2234. doi: 10.1182/blood-2002-01-0166
Peranteau, W. H., Heaton, T. E., Gu, Y. C., Volk, S. W., Bauer, T. R., and Alcorn, K. (2009). Haploidentical in utero hematopoietic cell transplantation improves phenotype and can induce tolerance for postnatal same-donor transplants in the canine leukocyte adhesion deficiency model. Biol. Blood Marrow Transplant. 15, 293-305. doi: 10.1016/j.bbmt.2008.11.034

Pirovano, S., Notarangelo, L. D., Malacarne, F., Mazzolari, E., Porta, F., Lanfranchi, A., et al. (2004). Reconstitution of T-cell compartment after in utero stem cell transplantation: analysis of T-cell repertoire and thymic output. Haematologica $89,450-461$.

Roncarolo, M. G., Yssel, H., Touraine, J. L., Betuel, H., De Vries, J. E., and Spits, H. (1988). Autoreactive T cell clones specific for class I and class II HLA antigens isolated from a human chimera. J. Exp. Med. 167, 1523-1534. doi: 10.1084/jem.167.5.1523

Sakaguchi, S., Sakaguchi, N., Asano, M., Itoh, M., and Toda, M. (1995). Immunologic self-tolerance maintained by activated $\mathrm{T}$ cells expressing IL-2 receptor alpha-chains (CD25). Breakdown of a single mechanism of self-tolerance causes various autoimmune diseases. J. Immunol. 155, 1151-1164.

Shaaban, A. F., Kim, H. B., Pameijer, C., Fichter, C., and Flake, A. W. (2000). Direct antigen presentation by donor cells in prenatal hematopoietic chimeras results in deletion of donor reactive $\mathrm{T}$ cell clones without $\mathrm{MHC}$ restriction. Transplantation 69(Suppl.), S241. doi: 10.1097/00007890-200004271-00500

Shields, L. E., Gaur, L., Delio, P., Potter, J., Sieverkropp, A., and Andrews, R. G. (2004). Fetal immune suppression as adjunctive therapy for in utero hematopoietic stem cell transplantation in nonhuman primates. Stem Cells 22, 759-769. doi: 10.1634/stemcells.22-5-759

Simonsen, M. (1955). The acquired immunity concept in kidney homotransplantations. Ann. N. Y. Acad. Sci. 58, 448-452. doi: 10.1111/j.1749-6632.1955.tb 45959.x

Sprent, J. (1995). Central tolerance of T cells. Int. Rev. Immunol. 13, 95-105. doi: $10.3109 / 08830189509061740$

Tanaka, S. A., Hiramatsu, T., Oshitomi, T., Imai, Y., and Koyanagi, H. (1998). Induction of donor-specific tolerance to cardiac xenografts in utero. J. Heart Lung Transplant. 17, 888-891.

Touraine, J. L., Raudrant, D., Golfier, F., Rebaud, A., Sembeil, R., Roncarolo, M. G., et al. (2004). Reappraisal of in utero stem cell transplantation based on long-term results. Fetal Diagn. Ther. 19, 305-312. doi: 10.1159/000077957

Touraine, J. L., Roncarolo, M. G., Raudrant, D., Bacchetta, R., Golfier, F., Sembeil, R., et al. (2005). Induction of transplantation tolerance in humans using fetal cell transplants. Transplant. Proc. 37, 65-66. doi: 10.1016/j.transproceed.2004. 12.006

Vrecenak, J. D., Pearson, E. G., Santore, M. T., Todorow, C. A., Li, H., Radu, A., et al. (2014). Stable long-term mixed hematopoietic chimerism achieved in a canine model of allogeneic in utero hematopoietic cell transplantation. Blood 124, 1987-1995. doi: 10.1182/blood-2013-11-537571

Wengler, G. S., Lanfranchi, A., Frusca, T., Verardi, R., Neva, A., Brugnoni, D., et al. (1996). In-utero transplantation of parental CD34 haematopoietic progenitor cells in a patient with X-linked severe combined immunodeficiency (SCIDXI). Lancet 348, 1484-1487. doi: 10.1016/S0140-6736(96)09 $392-0$

Conflict of Interest Statement: The author declares that the research was conducted in the absence of any commercial or financial relationships that could be construed as a potential conflict of interest.

Received: 29 September 2014; accepted: 28 October 2014; published online: 12 November 2014.

Citation: Peranteau WH (2014) In utero hematopoietic cell transplantation: induction of donor specific immune tolerance and postnatal transplants. Front. Pharmacol. 5:251. doi: 10.3389/fphar.2014.00251

This article was submitted to Integrative and Regenerative Pharmacology, a section of the journal Frontiers in Pharmacology.

Copyright (c) 2014 Peranteau. This is an open-access article distributed under the terms of the Creative Commons Attribution License (CC BY). The use, distribution or reproduction in other forums is permitted, provided the original author(s) or licensor are credited and that the original publication in this journal is cited, in accordance with accepted academic practice. No use, distribution or reproduction is permitted which does not comply with these terms. 